\title{
Evaluation of software tools supporting outcomes-based continuous program improvement processes: Part 3
}

\author{
Jake Kaupp and Brian Frank ${ }^{l}$ \\ Queen's University \\ jake.kaupp@queensu.ca
}

\begin{abstract}
The Canadian engineering accreditation board (CEAB) mandate tasked each engineering program to assess student outcomes in the form of graduate attributes and develop a data-informed continuous program improvement stemming from those assessments.

Administering, collecting and organizing the breadth assessment data is an extensive process, typically centralized through the use of software tools such as learning management systems (LMS), content management systems (CMS), Assessment Platforms (AP) and Curriculum Planning \& Mapping tools. These systems serve a variety of roles, ranging from course content delivery, e-learning, distance education, learning outcomes assessment, outcomes data management and learning outcomes analytics. Vendors have been developing various solutions to accommodate the shift towards outcomes based assessment as part of a continuous improvement process.

This paper will continue from the first and second papers presented at previous CEEA meetings. It will gauge how well each tool aligns with the EGAD (Engineering Graduate Attribute Development) project 5step process and compare and contrast software tools supporting outcomes based assessment as part of a continuous improvement process.
\end{abstract}

Keywords: Learning Management Systems, Assessment Management Systems, Curriculum Mapping, Academic Analytics, Data Management \& Reporting, Outcomes based assessment

\section{INTRODUCTION}

Canadian engineering programs are required to meet the Canadian Engineering Accreditation Board's mandate. Institutions must show that their graduates possess the outcomes representative of twelve graduate attributes, and must utilize the results of the outcomes assessment for the purposes of continual improvement of the program.

Engineering institutions are now quite familiar with creating and assessing student learning outcomes, curriculum mapping approaches and are now beginning to have some significant questions regarding how to best visualize, interpret and discuss the data. Many of these questions focus around the continuous improvement aspects of the $\mathrm{CEAB}$ mandate and are giving rise to some enlightened discussions regarding the use of learning analytics, information visualization and management principles from business and industry.

Central to all of these conversations is the wide variety of technologies that can assist in nearly every aspect of the process. The sheer number of the available tools is intimidating and innovated new tools are continually being released[1]. . This can lead to institutions selecting a tool based on reputation and popularity rather than considering the functionality of the tool and local climate and cultural influences towards technologies.

The EGAD Project has been raising the awareness of tools and technologies by providing evaluations of a number of tools and the strengths and weaknesses of each[2], [3]. The primary goals of these reviews is highlight that adopting a software tool or solution is not a quick process, and careful consideration and resources must be invested to ensure the successful, sustainable, long-term adoption of a software tool[4]. If the software tool is adopted without sufficient support, internal championing or consideration to change management issues, then it is unlikely to develop into a sustainable, long-term practice[5], [6].

This paper continues the theme of the first two installments: to present, compare and evaluate a variety of commercially and freely available software tools that support outcomes based assessment as part of a CPI process, reflected by the EGAD Projects 5-step approach. This installment looks at a variety of tools encompassing many classifications: Taskstream, Civitas Learning, Vena Solutions, CBlue and R.

\section{EVALUATION METHOD}

The vendors for each software tool were contacted and asked to deliver a presentation regarding the strengths and contributions of their particular software tool. Presentations were directed towards outcomes based assessment, data management, outcomes analytics and continuous program improvement capabilities. 
Following the presentations, the software tools were classified by 2 independent criteria and evaluated by 5 independent criteria, illustrated below. In the case of the new tools that do not incorporate the functionality, a N/A will be given for the select criteria. Additionally each tool is given a measure of how many facets of the EGAD 5-step approach the tool embodies.

\subsection{System Classification}

Each system is classified according to a typology outlined in Table 1 in the appendix, which provides a detailed description of each category.

\subsection{System Integration}

This evaluation criteria describes how the software tool is integrated with other educational technology, human resource systems and $3^{\text {rd }}$ party applications, as outlined in Table 2 in the appendix.

\subsection{Rubric-based Assessment}

This evaluation criteria focuses on the software tool's use of rubrics in assessment of student submissions. Ease of rubric creation, customization and storage for future use and sharing is considered, as outlined in Table 3 in the appendix.

\subsection{Learning Outcomes}

This evaluation criteria focuses on the software tool's use of student learning outcomes, outlined in Table 4 in the appendix. It evaluates at how learning outcomes can be created in an institution and if outcomes can be used at multiple levels (institution, faculty, department, course), if outcomes can be mapped across the levels (outcomes curriculum mapping), if outcomes can be linked to multiple assessment instances, and if outcomes can be archived into a searchable repository.

\subsection{Assessment}

This evaluation criteria focuses on the system's assessment capabilities, outlined in Table 5 in the appendix. It evaluates the types of student evidence used in assessment, the capability of multiple assessors on submitted evidence, the efficiency of grading student submissions, and the ease and quality of the feedback that can be provided to the student.

\subsection{Analytics}

This evaluation criteria focuses on the system's outcomes analytic capabilities, outlined in Table 6 in the appendix. It evaluates at the availability of reporting across institutional levels (down to student level), the flexibility of both tabular and graphical reporting, how on-demand the reporting methods are, the flexibility of longitudinal reporting methods and the ability to create custom groups (demographic or otherwise) for reporting.

\subsection{Pricing}

This evaluation criteria focuses on the price of the system. It evaluates the hosting model of the system, the duration of the subscription or licensing of the system, and the approximate cost of the system typically derived from full-time equivalent numbers.

\subsection{EGAD 5-step Alignment}

This evaluation element focuses on how many facets of the tool or solution align with the EGAD 5-step approach for outcomes based, data-informed, continuous improvement, outlined below:

1. Program Evaluation (Defining the key aspects, purposes and outcomes of your program)

2. Curriculum Mapping (Where are outcomes developed and assessed)

3. Assessment \& Data Collection

4. Analyzing and Interpreting Data (Reporting and analysis of the data)

5. Data informed Curriculum Improvement (Leveraging data to inform change)

Tools are awarded star per aligned aspect. It should be noted that achieving a high star ranking in this category does not imply that one tool is superior to another, as specialized solutions for a single facet of the EGAD 5step approach would possess a lower ranking than a more comprehensive tool that addresses multiple facets.

\section{SOFTWARE TOOL SUMMARIES}

In the interest of providing a rich evaluation of each software tool, a brief summary of each is provided below. These summaries focus on the particular strengths and weaknesses of the software tool that may not be evident from the evaluation criterion. 


\subsection{Taskstream}

Taskstream (http://www1.taskstream.com/) is a fully integrated assessment platform focused on advancing effective assessment for the purposes of improving student learning and institutional quality. It is designed to fully support the assessment cycle, offering support in planning, measurement, analysis and improvement. It offers users the ability to set goals, objectives and purpose, define outcomes and attributes, map the curriculum, assess student learning, report on results and develop plans for continuous improvement. The primary strengths of Taskstream are the vast utility of the platform that is rooted in effective practise, the support provided to clients, and the clear vision for continuous improvement.

Taskstream is separated into two key tools, the accountability management system and the learning achievement tool. These tools provide support for different aspects of assessment for continuous improvement, yet work synergistically to unify the oftenseparate student-level and program-level assessment.

The accountability management system provides support for multiple accreditation and quality related initiatives for programs, and steps users through a cohesive and logical flow (Mission, Outcomes, Maps, Plans, Findings, Actions). Review and approval is built into each step, with a check-out task system to plan and manage efforts typically done by committee. Outcomes can be created, stored, and searched across programs, or imported from popular accreditation frameworks (ABET, VALUE/DQP). Mapping is comprehensive with learning activity granularity. Reporting is done by static, usercreated reports with scalable views and can be modified to provide dashboard views. Results can be shared broadly with many stakeholders, and programs can generate their own accreditation report templates. Reviews and action plans can be created, easily linking assessment data to interpretation and then generating an action plan for approval.

The learning achievement tool is where the assessment of student learning takes place. Assessment in Taskstream is portfolio-based and rubric assessed. One of the interesting features in the Taskstream portfolio is that alignment is enforced, making sure that each aspect of the portfolio is assessed by a rubric which is linked to a specific set of outcomes mapped to the course. Assessment and feedback is quickly given by in-line touch-based assessment, which can facilitate multiple assessment models (holistic/analytic rubrics, criterion/norm based assessment, meta-rubric assessment via VALUE rubrics). Reporting is presented similarly to that in the accountability system, allowing instructors to see assessment results in an on demand fashion, and the ability to customize their dashboards for at-a-glance information.
The main weakness of Taskstream is the two stage nature of the platform. It is not a learning management system, and does not incorporate the more traditional means of assessment in engineering. While it is well integrated, students must still submit work or aspects of their portfolio through the LMS to Taskstream. This 2stage nature is a common drawback in assessment platforms making them less attractive to institutions seeking to reduce their technology footprint and reduce burden on students often flooded with multiple systems for learning and assessment. Overall, Taskstream is very well aligned with all facets of the EGAD 5-step approach receiving full value for this category. It offers institutions a modern, effective-practise based solution designed for cyclical and continuous improvement of student learning and program quality.

\subsection{Civitas Learning}

Civitas Learning (http://www.civitaslearning.com/) is a relatively new platform and is an analytics platform designed to help with student success. It is a modern, cloud-based system that uses predictive analytics and modern HTML5 web-applications to provide actionable information and personalized recommendations to students, faculty and student affairs professionals. Currently, Civitas Learning is largely focused on retention and student persistence aspects of student success, with plans to incorporate predictive models of student success. It looks to leverage historical and current data, as well as diverse factors and predictors and measures of engagement at the course, student and program levels by unifying data systems and sources (LMS, SIS, Planning \& Curriculum software). The primary strengths of Civitas Learning is its reporting and analytics power and its Degree Map tool which represents common paths through programs in a manner easily understood by students and administration.

Civitas Learning is separated into a key platform and application suites. Illume is the primary predictive analytics and reporting platform which provides views of historical performance and predicted success. It is also an application that helps provide the primary information to student success presented in the form of "course signals". Civitas Learning also provides the Degree Map and Inspire application suites, which provide students with formative information regarding degree choices and pathways and offer real-time information to help support student success and course completion.

The Illume platform and application runs an institution and population specific predictive model, developed in consultation with Civitas Learning specialist upon adoption of the platform. It provides flexible and customizable dashboards that convey real-time information to stakeholders, which can be scaled to many levels of granularity or to a single student. As previously 
mentioned the information presented by Illume focuses largely on persistence in a course or program, and can alert stakeholders when a student is predicted to withdraw from a program and offer insights into intervention strategies. These interventions can also be automated to provide suggestions to cohorts, based on typical performance (e.g. study strategies, targeted support). It offers a unique perspective for course personnel and student affairs to see patterns of student engagement in courses and see what predictors most influence students to persists.

The Degree Map and Inspire application suites rely on the Illume platform for information, but present distinctly separate information. Degree Map provides students and administration with discrete pathways for degrees that are attentive to the student's previous and predicted performance. The student is provided with information for alternate degrees that they would have a greater likelihood to complete. It offers the ability for students and advisors to see degree changes and the likelihood of success, what would be required and what credits needed to complete the degree, and the associated tuition costs. Inspire provides administration and student views primarily related to course-based engagement measures and the risk of students dropping the course. It allows administrators to quickly view groups and cohorts and send out personalized interventions and communications to help those students re-engage and overcome their particular adversity.

The main weakness of Civitas Learning is the narrow focus on student persistence and engagement. This isn't a great issue in the Canadian university system, may have considerable applications in the college system. However, establishing predictive measures for these should not be taken lightly and is a considerable feat of data science, cognitive sciences, and engineering. What would be beneficial is the ability to create a predictive model for student success, performance or attainment of learning outcomes, attributes or competencies. This could be based on historical performance drawn from assessment information alongside the other elements of Illumes predictive model. Overall, Civitas Learning achieved a 3 star rating for alignment with the EGAD 5 step approach being well aligned with the first, second and fourth step of the process.

\subsection{VENA Solutions}

Vena Solutions (http://venasolutions.com/) is a corporate performance management solution that has recently ventured into the accreditation arena. Vena is a quality, performance and process management solution that leverages the familiar tools and processes already in place at many companies and institutions. Vena integrates into excel worksheets and books and provides a common regulated and verified process to unify and streamline data collection. It also provides flexible reporting and analysis, powered by a proprietary BI (business intelligence) tool. The focus of Vena is to quickly and efficiently have users populate a database with the required information using an approach they are familiar and comfortable with, but adds layers of process and quality control (check-in/check-out systems, common workflows). Vena focuses on the process with a large focus on continuous improvement, and how users can provide input and feedback on their submitted data and that of others.

The overall workflow for an accreditation process within Vena is the creation of a learning plan (outcomes and mapping), grade inputs (assessment data), recommendations (reporting and process input) and reviews (actions and improvements). Each of these items is attached to a process, which is typically categorized by years within the process. Within each of these elements are different aspects of the accreditation process. Users initially see a task-oriented view of the process, which outlines to them their required work and objectives to move the process forward.

In the learning plan, Vena provides the ability to create custom objectives and outcomes structures for institutions and programs, each with accountability and review built in. This allows for the creation of draft outcomes and work in progress, which allows for instructors to create and amend outcomes as they see fit. Accessibility, review and approval is role-based, with only selected users being able to approve items. The check-in/check-out workflow restricts duplication of work and unnecessary or accidental modification of data. The second aspect of the learning plan is curriculum mapping. Vena supports curriculum mapping by a variety of userdefined styles as they adhere to the standard matrix-style mapping approach. Mapping is connected to the assessment level, which also allows instructors to apply weighting to the results.

Assessment data is entered via the excel interface, and is typically recorded by a rubric criterion level and not a grade. However, this could be easily customized as Vena can utilize any excel functionality in a seamless manner. Results are presented quickly and readily in variety of easily customizable reports and visualizations as Vena accommodates just-in-time reporting capabilities, and leverages the customization inherent in excel graphing.

The key strength of Vena is the recommendations and reviewing, which places great emphasis on a collaborative improvement process with data and front-line input at the forefront. Vena also realizes that each individual process needs to be tailored to suit diverse needs of intuitions and include a process planner (similar to VISIO) that allows users to plan their approach to meet specific needs and requirements, incorporate elements to facilitate reflection and build a rigorous process with pre-defined review elements and checkpoints. 
The primary weakness of Vena is that it is distinctly separate from the overall assessment process. This is the same challenge facing many assessment platforms, that it is one more tool that has to be used within a process. Depending upon the intended future functionality of the platform, this may require a duplication of grading/assessment for individual instructors. The LMS is the typical grade repository with either marks being directly entered or uploaded to it. Adding results to Vena is another additional step, which may cause issues. There are also minor concerns about the sustainability of an excel based process that presents a non-platform agnostic interface. Overall Vena achieved a 5 star rating, representing a well deal aligned with the EGAD 5-step process.

\subsection{CBlue}

CBlue, or Curriculum Blueprint (http://www.cbluesoftware.com/) began as a curriculum mapping tool developed for the School of Medicine at Memorial University. It is now a commercially available product that focuses on curriculum development and mapping for program level, competency-based assessment. It incorporates a flexible design for courses and mapping can be conducted at a very fine grain with many possible relationship combinations (e.g. multiple outcomes/competencies in multiple areas). It also supports unique meta-data and accreditation related tagging that allows users to specific collect and report on unique or distinctive aspects of curriculum.

CBlue presented users with 3 primary views. The first, big picture view; provides users with a high-level overview of curriculum. This provides users with a quick means of determining the scope and sequence of curriculum, suitable for a general audience to get a "feel" of a program. The second view of CBlue is integrated with the LMS, and is intended to provide the student with a middle-level granular view of curriculum, and connect their courses and learning experiences and see how each course contributes to obtaining competencies/outcomes. The final view is the administration view, which is the very granular "nuts and bolts" view of the curriculum, intended for those with a deep understanding of curriculum development and provide the means to manage and change the curriculum outside the CBlue system.

CBlue can accommodate a number of program level competencies and outcomes structures, and each can be stored in a fully searchable repository. Multiple competency or outcomes sets within a single program are easily handled. Curriculum can be developed in a leveled capacity with each respective years curriculum progressing to the next. CBlue also incorporates a learning objectives wizard to help develop objectives according to Miller's pyramid. While these are not learning outcomes per se, the tool can be used to help faculty new to the area have a starting point to develop their own course learning outcomes.

CBlue allows for highly flexible mapping options, linking program outcomes to courses, lessons, learning activities, assessments, or contextual elements (interviews, simulations, etc.). The tool helps to foster constructive alignment through session planning. Instructors or developers can build sessions, add teaching and learning methods, assessment methods, map to program level outcomes and then related those to course objectives and outcomes. Reporting on the curriculum is currently in development with only a limited number of reports available (gap analysis, accreditation reports).

The weaknesses of CBlue are the stand-alone nature of the tool, and the lack of meaningful reporting. CBlue is somewhat integrated into an LMS but it was unclear whether or not the system can create outcomes, and apply mappings within courses or learning events within the LMS. Also it was unclear if the system could pull the existing curriculum and outcomes information from the LMS and integrate those into a skeleton of a new blueprint within CBlue. The reporting was specified as "in development" with the potential for an expanded and improved set of reporting in the near future.

Overall, Atlas obtained a three star rating demonstrating alignment with three of the five steps of the EGAD approach. This is primarily due to the specific focus of the tool limits its use in assessment (beyond planning) and in the analysis and interpretation of the assessment data.

\section{$3.5 \mathrm{R}$}

The more controversial and unexpected tool to include in this review is the open-source statistical computing language $\mathrm{R}$ (http://www.r-project.org/). $\mathrm{R}$ is in no way an LMS, Assessment Platform or any typical piece of educational technology. $\mathrm{R}$ is an implementation of the $\mathrm{S}$ language invented in Bell Labs in 1976. It is an open source integrated suite of software facilities for data manipulation, calculation and graphics. It is often described as a programming language, but developers see it more as an environment in which statistical procedures are applied[7]Team:2000tp\}. It is fully extensible and open source, with 7393 distinct packages released thus far, with countless more in development. It is capable of leveraging and invoking many popular programming languages $(\mathrm{C}, \mathrm{C}++)$, to rival speed and power of many commercial packages. One of the underlying goals of the $\mathrm{R}$ Project was the production of high quality graphics and visualizations in which the user has complete control.

In conjunction with RStudio (http://www.rstudio.com) an integrated development environment, and many packages developed by the makers of RStudio, $\mathrm{R}$ can serve as a powerful analytics, reporting and data 
visualization platform for a wide variety of assessment data.

$\mathrm{R}$ can read data directly from many formats or even communicate and pull data from a database or even be configured to pull directly from an API. Once data is populated, users can create automated scripts to perform most basic and advanced analysis tasks. Once created these scripts can be customized and manipulated to perform ad-hoc reporting and answer a wide variety of data-related questions. Particularly interesting are the packages: tidyr, reshape2, dplyr, magrittr, ggvis which form the basis for the collection, manipulation and visualization of data in a highly customizable, quick and reproducible workflow[8].

These tools can be supplemented by knitr and rmarkdown packages, which leverage lightweight writing languages (rmarkdown) and typesetting and conversion languages (pandoc) to author reproducible reports that can include high quality graphics produced by $\mathrm{R}$. These reports can also be interactive documents, with the help of the shiny package, which can be published to the web to allow users to interact with static views of data presented in the reports.

Even more promising is the continued development of the shiny package into a framework for the development of applications directly from $\mathrm{R}$ that can be used for the indepth analysis, exploration and visualization of data. Parallel to the development of shiny, the RStudio team developed a server application for shiny, complete with a number of implementation options (SAAS open-source self-hosted, or a professional installation on a local server). This approach would allow for an institution to develop it's own approach for data analysis and visualization and share the results broadly with a variety of stakeholders. Security concerns are easily addressed by the professional installation of shiny, on a institution controlled server, complete with encryption and a host of authentication options. Recently, shiny application package called radiant was developed, which provides users with the ability to fully explore and visualize a dataset of their choice with a large host of options.

Everything done within $\mathrm{R}$ and the packaged developed by the community are completely open-source and customizable. A great deal of visualization packages in $\mathrm{R}$ are exploring ways to utilize many of the popular JavaScript frameworks for data visualization ( $\mathrm{d} 3 \mathrm{j}$, rickshaw, highcharts, charts, sigma) and analysis, which vastly enhances the functionality of the platform and improves the ability to produce high-quality, modern, web capable interactive depictions of assessment data.

Despite all of the freedom, extensibility and opensource aspects of $\mathrm{R}$, there are still drawbacks. The main challenges of $\mathrm{R}$ are experience, sustainability, and scale. Significant experience in coding, and significant exploration in the language is required to produce some of the more complex visualizations and applications and to explore and leverage the capabilities of an ever-expanding list of packages. Concerns regarding scale and sustainability are faced by any open-source or user developed process. Using $\mathrm{R}$, while attractive at start, requires considerable investment in expertise, infrastructure, development and maintenance. All of these costs should be carefully considered and factored into any decision. A more sustainable use may be using $\mathrm{R}$ for ad-ho, just-in-time, just-for-me analysis in concert with an integrated LMS and Analytics system that can provide answers and analysis for more standard questions. This approach does provide the option to leverage, analyze and report on external assessment initiatives running at an institution (e.g. NSSE, CLASSE, VALUE, etc.)

Overall, $\mathrm{R}$ is a suitable tool, but only received a ranking of 3 stars with respect to the EGAD 5-step process, due to the lack of assessment and curriculum mapping capability.

\section{EVALUATION RESULTS}

Each software tool was classified and evaluated for each criterion and the results tabulated into the table shown in Figure 1 in the appendix.

\section{CONCLUSIONS}

Similar to the previous two installments of this paper, it should be noted that the evaluations presented are not intended to objectively rank or promote the use of a single tool. The purpose of these reviews is to offer a brief review of the tool and its purpose and highlight its strengths and weaknesses. All of the evaluated solutions are capable of being an integral part of an outcomesbased, data-informed continuous improvement process.

With the recent and quick rise of analytics as a potential solution to a number of problems in higher education, institutions must carefully consider the adoption of technology into their unique culture. Technologies should be carefully evaluated and scrutinized before adopting, rather than choosing a solution in haste. This is an issue in change management and institutions should carefully consider a variety of factors before adopting a new tool or solution as part of their process[9]. To reiterate from the previous papers[2], [3], such factors include, but are not limited to[5], [10], [11]:

1. Stakeholder needs and requirements

2. Direction and leadership of CPI processes

3. Existing climate regarding new technology

4. Complexity \& sustainability of tools 


\section{Acknowledgements}

The authors would like to acknowledge support from the DuPont Canada Chair in Engineering Education Research and Development. Support for the EGAD initiative was provided by Engineers Canada and the National Council of Deans of Engineering and Applied Science.

\section{References}

[1] D. McIntosh, "Vendors of learning management and e-learning products," 2014.

[2] J. A. Kaupp, B. Frank, and C. Watts, "Evaluation of software tools supporting outcomes-based continuous program improvement processes," presented at the Proceedings of the Canadian Engineering Education Association, Montreal, QC, 2013.

[3] J. A. Kaupp and B. Frank, "Evaluation of software tools supporting outcomes-based continuous program improvement processes Part 2," presented at the Proceedings of the Canadian Engineering Education Association, Canmore, AB, 2014, pp. 1-10.

[4] E. Heinrich, J. Milne, A. Ramsay, and D. Morrison, "Recommendations for the use of e-tools for improvements around assignment marking quality," Assessment \& Evaluation in Higher Education, vol. 34, no. 4, pp. 469-479, Aug. 2009.

[5] G. R. Baker, N. A. Jankowski, S. Provezis, and J. Kinzie, "Using Assessment Results: Promising Practices of Institutions That Do It Well," National Institute for Learning Outcomes Assessment, 2012.

[6] T. W. Banta, E. A. Jones, and K. E. Black, Designing Effective Assessment: Principles and Profiles of Good Practice. John Wiley $\backslash \&$ Sons, 2009.

[7] R. Ihaka and R. Gentleman, "R: A Language for Data Analysis and Graphics," Journal of Computational and Graphical Statistics, vol. 5, no. 3, pp. 299-314, Feb. 2012.

[8] H. Wickham, "Big data pipelines," presented at the Workshop on Visualization for Big Data Strategies and Principles, Toronto, 2015.

[9] G. Scott, "Effective change management in higher education," Educause review, vol. 38, pp. 64-78, 2003.

[10] C. F. Blaich and K. Wise, "From Gathering to Using Assessment Results," National Institute for Learning Outcomes Assessment, 2011.

[11] A. L. McCann, "Factors affecting the adoption of an e-assessment system," Assessment \& Evaluation in Higher Education, vol. 35, no. 7, pp. 799-818, Dec. 2010. 


\begin{tabular}{|l|c|c|c|c|c|}
\cline { 2 - 6 } & Taskstream & $\begin{array}{c}\text { Civitas } \\
\text { Learning }\end{array}$ & Vena & CBlue & R \\
\hline 1. Classification & AP & AS & AS & CMT & AS \\
\hline \hline 2. Integration & LTI \& API & LTI \& API & API & API & API \\
\hline \hline
\end{tabular}

\section{Rubric-based assessment}

\begin{tabular}{|c|c|c|c|c|c|c|}
\hline $3 a$. & Rubric Generation & $\hat{\alpha} \hat{\alpha} \hat{z}$ & $\mathrm{~N} / \mathrm{A}$ & $\mathrm{N} / \mathrm{A}$ & $\mathrm{N} / \mathrm{A}$ & $\mathrm{N} / \mathrm{A}$ \\
\hline $3 b$. & Customizable & 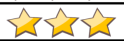 & $\mathrm{N} / \mathrm{A}$ & $\mathrm{N} / \mathrm{A}$ & $\mathrm{N} / \mathrm{A}$ & $\mathrm{N} / \mathrm{A}$ \\
\hline $3 c$ & Rubric Repository & 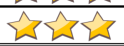 & $\mathrm{N} / \mathrm{A}$ & $\mathrm{N} / \mathrm{A}$ & $\mathrm{N} / \mathrm{A}$ & $\mathrm{N} / \mathrm{A}$ \\
\hline
\end{tabular}

\begin{tabular}{|c|c|c|c|c|c|c|}
\hline \multicolumn{7}{|c|}{ 4. Learning Outcomes } \\
\hline 4a. & Multi-level capability & $\hat{\mathrm{a}} \hat{\mathrm{L}} \hat{\mathrm{U}}$ & 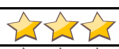 & $\hat{\mathrm{U}} \hat{\mathrm{Z}} \hat{\mathrm{U}}$ & 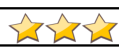 & $\mathrm{N} / \mathrm{A}$ \\
\hline 4b. & Multi-level mapping & 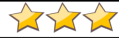 & 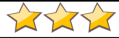 &  & सेत्रित्र & $\mathrm{N} / \mathrm{A}$ \\
\hline 4c. & Multi-instance mapping & $\hat{\boldsymbol{W}} \hat{\mathrm{W}} \hat{\mathrm{W}}$ & $\hat{\imath} \hat{A} \hat{Z}$ & $\hat{\mathrm{L}} \hat{\mathrm{U}}$ & 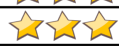 & $\mathrm{N} / \mathrm{A}$ \\
\hline 4d. & Outcomes Repository & 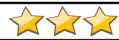 & 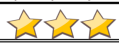 & $\hat{\vec{n}}$ & $\hat{厶} \hat{厶}$ & $\mathrm{~N} / \mathrm{A}$ \\
\hline
\end{tabular}

\begin{tabular}{|c|c|c|c|c|c|c|}
\hline \multicolumn{7}{|c|}{ 5. Assessment } \\
\hline $5 a$. & Direct \& Indirect Evidence & $\hat{\mathcal{A}} \tilde{\hat{n}}$ & $\mathrm{~N} / \mathrm{A}$ & $\mathrm{N} / \mathrm{A}$ & $\mathrm{N} / \mathrm{A}$ & N/A \\
\hline $5 b$. & Multiple assessors &  & $\mathrm{N} / \mathrm{A}$ & $\mathrm{N} / \mathrm{A}$ & $\mathrm{N} / \mathrm{A}$ & $\mathrm{N} / \mathrm{A}$ \\
\hline $5 c$. & In-line grading & 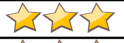 & $\mathrm{N} / \mathrm{A}$ & $\mathrm{N} / \mathrm{A}$ & $\mathrm{N} / \mathrm{A}$ & N/A \\
\hline $5 d$. & In-line feedback & $\hat{\mathrm{W}} \hat{\mathrm{Z}}$ & $\mathrm{N} / \mathrm{A}$ & $\mathrm{N} / \mathrm{A}$ & $\mathrm{N} / \mathrm{A}$ & $\mathrm{N} / \mathrm{A}$ \\
\hline
\end{tabular}

6. Analytics

\begin{tabular}{|c|c|c|c|c|c|c|}
\hline 6a. & Multi-level reporting & $\hat{\omega} \hat{W} \hat{L}$ & $\hat{\omega} \hat{W}$ & $\hat{\omega} \hat{\omega} \hat{n}$ & $\hat{\omega} \hat{n} \hat{n}$ & $\hat{\mathcal{A}} \hat{\imath}$ \\
\hline $6 b$. & Tabular reporting & $\hat{\tilde{L}} \hat{\imath}$ & 可在 & $\hat{\omega} \hat{\imath}$ & $\hat{厶} \hat{W}$ & $\hat{\omega} \hat{\imath} \hat{y}$ \\
\hline 6c. & Graphical reporting & $\hat{\underline{W}}$ & $\hat{\mathrm{L}} \hat{\mathrm{n}}$ & $\hat{W} \hat{W}$ & $\hat{L}$ & क्रिय \\
\hline $6 d$. & On-demand reporting & $\hat{\omega} \hat{1} \hat{L}$ & 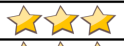 & $\hat{\omega} \hat{U} \hat{U}$ & 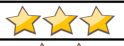 & 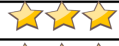 \\
\hline $6 e$. & Longitudinal reporting & $\hat{\omega} \hat{厶}$ & 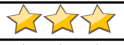 & $\hat{\omega} \hat{W} \hat{H}$ & $\hat{厶} \hat{\imath}$ & $\hat{\Sigma} \hat{\imath}$ \\
\hline $6 f$. & Custom group reporting & 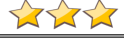 & सेखि口 & $\hat{\mathrm{W}} \hat{\mathrm{W}}$ & $\hat{n}$ & पूरिद्र \\
\hline
\end{tabular}

\section{Pricing}

\begin{tabular}{c|l|c|c|c|c|c|}
\hline 7a. & Hosting Model & SaaS & SaaS & Self & Self & Self or SaaS \\
\cline { 2 - 6 } 7b. & Subscription & Yearly License & Yearly License & Yearly License & Yearly License & Open Source \\
\cline { 2 - 6 } 7c. & Cost & Flexible options & Flexible options & Inquire & Inquire & Flexible options \\
\hline \hline
\end{tabular}

\section{EGAD 5-Step Alignment}

\begin{tabular}{|c|c|c|c|c|}
\hline 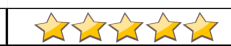 & 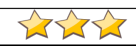 & 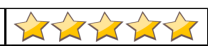 & $\hat{\imath} \hat{\imath} \hat{\imath}$ &  \\
\hline
\end{tabular}

Figure 1 - Evaluation results of software tools 